\section{REVISTA BRASILEIRA DE QUALIDADE DE VIDA}

\title{
Inferências da Organização Mundial da Saúde na promoção da qualidade de vida: uma explanação contemporânea dos instrumentos WHOQOL
}

\section{Inferences of the World Health Organization in quality of life promotion: a contemporary explanation of the WHOQOL instruments}

\author{
Bruno Pedroso \\ Universidade Estadual de Ponta Grossa - UEPG - Ponta Grossa - Brasil \\ brunops3@brturbo.com.br \\ Claudia Tania Picinin \\ Universidade Tecnológica Federal do Paraná - UTFPR - Ponta Grossa - Brasil \\ claudiapicinin_adm@yahoo.com.br \\ José Roberto Herrera Cantorani \\ Universidade Estadual de Campinas - UNICAMP - Campinas - Brasil \\ cantorani@yahoo.com.br \\ Gustavo Luis Gutierrez \\ Universidade Estadual de Campinas - UNICAMP - Campinas - Brasil \\ glgutierrez@terra.com.br
}

\section{RESUMO}

OBJETIVO: O presente estudo tem como objetivo identificar os diferentes instrumentos WHOQOL existentes na literatura e também suas estruturas e propósitos.

MÉTODOS: Trata-se de um estudo exploratório de natureza bibliográfica.

RESULTADOS: Constatou-se que a partir do projeto inicial, que resultou no WHOQOL-100, um instrumento genérico, a Organização Mundial da Saúde ampliou seus objetivos e deu início a projetos para novos instrumentos, voltados para a avaliação da qualidade de vida de grupos específicos. Também estruturou uma versão abreviada do instrumento original. E o mesmo foi feito para alguns instrumentos de caráter específico.

CONCLUSÕES: O WHOQOL-100 se mostrou um instrumento muito eficaz na avaliação da qualidade de vida de forma geral. Por isso este foi composto por facetas que representam os diferentes domínios da vida. No entanto, em razão de sua estrutura genérica, não se constitui em um instrumento com grande eficácia para grupos específicos. Ciente deste fato, a Organização Mundial da Saúde desenvolveu instrumentos para alcançar eficiência na leitura da qualidade de vida de crianças, idosos, pessoas soropositivas, pessoas com deficiência e pessoas com dor crônica.

PALAVRAS-CHAVE: Qualidade de vida. Organização Mundial da Saúde. WHOQOL. 


\section{ABSTRACT}

OBJECTIVE: The present study objectifies to identify the different WHOQOL instruments presents in literature and also its structures and purposes.

METHODS: It is an exploratory and bibliographical research.

RESULTS: We verified that from the initial project, that resulted in WHOQOL-100, a generic instrument, the World Health Organization enlarged its objective and started to project new instruments, addressed to the quality of life assessment of specific groups. It also structured an abbreviated version of the original instrument. It was also done to some specific instruments.

CONCLUSIONS: WHOQOL-100 showed a very effective instrument in quality of life assessment in a general form. Therefore, it was composed by facets that represent different domains of the life. However, due its generic structure, it was not an instrument with great effectiveness for specific groups. Aware of this fact, World Health Organization developed instruments to reach efficiency in quality of life assessment of children, older people, people living with HIV, people with disability and people with chronic pain.

KEYWORDS: Quality of Life. World Health Organization. WHOQOL.

\section{Introdução}

Não obstante à existência de diversos instrumentos de avaliação da qualidade de vida (QV), é sabido que a maior parte destes são específicos, avaliando tal variável em função de um aspecto particular. Em vista da carência de um instrumento que avaliasse a QV de forma global, a Organização Mundial da Saúde (OMS) empenhou-se na construção do Instrumento de Avaliação da Qualidade de Vida da Organização Mundial da Saúde (WHOQOL).

O ponto de partida para a construção do WHOQOL foi conceituar o termo QV, sendo esta entendida como "a percepção do indivíduo de sua posição na vida no contexto da cultura e sistema de valores nos quais ele vive e em relação aos seus objetivos, expectativas, padrões e preocupações" (FLECK et al., 1999, p. 20).

Frente a esse conceito, a OMS inicia o projeto WHOQOL em 1993. O sucesso do instrumento progenitor, o WHOQOL-100, instigou a OMS a construir diversos outros instrumentos sob tal metodologia. O presente estudo objetiva identificar os diferentes instrumentos WHOQOL existentes na literatura e também suas estruturas e propósitos.

\section{Projeto WHOQOL}

O desenvolvimento do WHOQOL teve início em 1994 e foi realizado em 15 centros simultaneamente, sediados em 14 países. Após o desenvolvimento do projeto WHOQOL, novos centros foram incorporados e o WHOQOL-100 foi disponibilizado em mais de 20 idiomas. A metodologia de desenvolvimento do WHOQOL foi seccionada em quatro grandes etapas: clarificação do conceito de QV, estudo piloto qualitativo, desenvolvimento de um piloto e uma aplicação de campo. Após a conclusão da elaboração do instrumento, foi estabelecida uma metodologia que consistia na sua tradução, preparação do teste piloto, desenvolvimento das escalas de respostas e administração do piloto (GRUPO WHOQOL, 1998).

A partir das sugestões formuladas por cada um dos centros de desenvolvimento, totalizou-se aproximadamente 1800 questões. Destas foram selecionadas 235 questões, seccionadas em 29 facetas, para o instrumento piloto do WHOQOL-100. Após a aplicação do teste piloto, foram escolhidas as melhores questões para cada faceta, de forma a estabelecer a consistência interna e validade discriminante do instrumento. Foram selecionadas 100 questões seccionadas em 24 facetas. As facetas foram agrupadas em seis grandes domínios (FLECK et al., 1999).

O WHOQOL-100 é seccionado em 24 grupos de quatro questões cada, recebendo a denominação de "facetas". Por sua vez, o conjunto de facetas constitui um "domínio". Ao contrário 
da composição das facetas, os seis domínios do WHOQOL-100 não são constituídos pelo mesmo número de facetas, podendo variar de uma até oito facetas. Há ainda uma faceta que não está inserida em nenhum domínio. Esta faceta, nomeada "Qualidade de vida global e percepção geral da saúde", aborda uma auto avaliação da QV, em que o indivíduo expressa o seu ponto de vista de satisfação com a sua vida, saúde e QV.

As questões que compõem o WHOQOL-100 não estão dispostas no questionário seguindo uma sequência lógica por domínio ou por faceta, mas sim, por tipo de escala de respostas. Os domínios do WHOQOL-100 são: Físico, composto pelas facetas Dor e desconforto, Energia e fadiga e Sono e repouso; Psicológico, composto pelas facetas Sentimentos positivos, Pensar, aprender, memória e concentração, Autoestima, Imagem corporal e aparência e Sentimentos negativos; Nível de independência, composto pelas facetas Mobilidade, Atividades da vida cotidiana, Dependência de medicação ou de tratamentos e Capacidade de trabalho; Relações sociais, composto pelas facetas Relações pessoais, Suporte social e Atividade sexual; Ambiente, composto pelas facetas Segurança física e proteção, Ambiente no lar, Recursos financeiros, Cuidados de saúde e sociais, Oportunidades de adquirir novas informações e habilidades, Participação em, e oportunidades de recreação/lazer, Ambiente físico e Transporte e; Aspectos espirituais/Religião/Crenças pessoais, composto pela faceta Espiritualidade/religião/crenças pessoais.

Todas as questões do WHOQOL-100 são fechadas. É utilizada uma escala de respostas do tipo Likert de cinco pontos, variando entre 1 e 5. Esses extremos representam 0\% e 100\%, respectivamente. Existem quatro tipos diferentes de escala de respostas: intensidade, avaliação, capacidade e frequência.

Tendo por base o WHOQOL-100, posteriormente foram construídos outros instrumentos de avaliação da QV. Com o objetivo de disponibilizar um instrumento que demande menor tempo para preenchimento e com características psicométricas satisfatórias, o Grupo WHOQOL desenvolveu a versão abreviada do WHOQOL-100, o WHOQOL-bref.

O WHOQOL-bref é composto por 26 questões, sendo duas questões sobre a auto avaliação da QV e 24 questões representando cada uma das facetas do WHOQOL-100. Para a composição das questões do WHOQOL-bref foi selecionada a questão de cada faceta que apresentava a maior correlação com o escore médio de todas as facetas (THE WHOQOL GROUP, 1996).

Após a seleção das questões, foi realizada uma análise para verificar se estas, de fato, representavam as facetas correspondentes. Em seis facetas a questão selecionada foi substituída por outra questão da faceta correspondente, pois, sob o viés de especialista, havia outra questão que melhor definisse essas seis facetas (THE WHOQOL GROUP, 1996). As facetas pertencentes ao domínio Nível de independência foram incorporadas ao domínio Físico, assim como a faceta pertencente ao domínio Aspectos espirituais/Religião/Crenças pessoais foi incorporada ao domínio Psicológico. Assim, o WHOQOL-bref é composto por quatro domínios: Físico, Psicológico, Relações sociais e Meio-ambiente.

No intento de construir um instrumento de avaliação da QV para o Projeto EUROHIS, cujo objetivo era a elaboração de questionários e entrevistas para avaliação de indicadores da área da saúde no continente europeu, fora convencionado que se tentaria adaptar um instrumento já existente para uma versão com um pequeno número de questões, para utilização em pesquisas em nível nacional, tais quais os censos demográficos (POWER, 2003).

O EUROHIS-QOL, posteriormente batizado de WHOQOL-8, é composto por oito questões derivadas empiricamente do WHOQOL-bref, sendo considerado uma alternativa viável para utilização em grandes populações ou em situações em que se demande de pouco tempo para o preenchimento do questionário (AUSTRALIAN WHOQOL FIELD CENTRE, 2006).

\section{Prossecução e instrumentos específicos}

Com o intuito de criar um instrumento de avaliação da QV direcionado para portadores do HIV, pesquisadores da UNAIDS e da OMS realizaram estudos em portadores do HIV em nove 
países. O resultado desse estudo foi o instrumento WHOQOL-HIV, um suplemento ao WHOQOL100 específico para pessoas portadoras do HIV ou AIDS.

O WHOQOL-HIV avalia a QV a partir de seis domínios e 29 facetas. Os domínios e as facetas são as mesmas do WHOQOL-100, com o acréscimo de cinco facetas específicas para portadores do HIV. As facetas específicas para os portadores do HIV, assim como as facetas do WHOQOL-100, são compostas por quatro questões (O'CONNELL et al., 2004).

As facetas adicionais do WHOQOL-HIV se inserem nos domínios já existentes do WHOQOL-100, sendo o Domínio Físico acrescido da faceta Sintomas de pessoas vivendo com HIV/AIDS (PVHAs), o Domínio Psicológico acrescido da faceta Inclusão social e o Domínio Aspectos espirituais/Religião/Crenças pessoais acrescido das facetas Perdão e culpa, Preocupações sobre o futuro e Morte e morrer.

Sob a mesma justificativa da elaboração do WHOQOL-bref, o Grupo WHOQOL desenvolveu uma versão abreviada do WHOQOL-HIV. O WHOQOL-HIV-bref é baseado no WHOQOL-bref, da forma que cada faceta é representada por uma única questão, somado a duas questões gerais da QV. As 26 questões do WHOQOL-bref se repetem no WHOQOL-HIV-bref, sendo estas acrescidas de cinco questões que representam as facetas adicionais do WHOQOL-HIV (THE WHOQOL-HIV GROUP, 2002).

Ao contrário do que ocorre no WHOQOL-bref, as facetas pertencentes aos domínios nível de independência e Aspectos espirituais/Religião/Crenças pessoais não são incorporadas aos domínios Físico e Psicológico, assumindo, assim, a mesma configuração dos domínios presentes no WHOQOL-100 e WHOQOL-HIV.

Tendo em vista que algumas questões dos instrumentos WHOQOL-100 e WHOQOL-bref não se aplicam às crianças e/ou influenciam a QV destas por meio de outro foco, surge a proposta da construção de uma versão dos instrumentos WHOQOL com direcionamento para crianças. Inicialmente batizado como C-QOL, tendo em vista a sua derivação do WHOQOL-100, posteriormente recebeu a denominação de WHOQOL-Children (LUZNY, 2011).

O WHOQOL-Children contempla os seis domínios presentes no WHOQOL-100, acrescido do domínio adicional Direitos políticos das crianças. As facetas que constituem os domínios já existentes no WHOQOL-100 são as mesmas, com exceção da faceta Dependência de drogas, que foi acrescida ao domínio Nível de independência e da faceta Atividade sexual, que foi excluída do domínio Relações sociais. Com relação ao domínio adicional do WHOQOL-Children, as facetas que compõem esse domínio são Direito de falar e ser ouvido e Direito à identidade e cidadania.

Cada faceta é representada por duas questões, totalizando 54 questões. Ao contrário dos demais instrumentos WHOQOL, as questões do WHOQOL-Children estão agrupadas por faceta e domínio (JIROJANAKUL; SKEVINGTON; HUDSON, 2003).

Almejando adaptar o WHOQOL para a aplicação em adultos idosos, o Grupo WHOQOL desenvolveu um módulo adicional denominado WHOQOL-OLD. Ao contrário do WHOQOL-HIV e WHOQOL-HIV-bref, o WHOQOL-OLD não suplementa o WHOQOL-100 ou o WHOQOL-bref. O WHOQOL-OLD é um módulo complementar, mas que deve ser aplicado em conjunto com o WHOQOL-100 ou o WHOQOL-bref (POWER et al., 2005).

O WHOQOL-OLD é composto por 24 questões seccionadas em seis facetas, formando um domínio adicional questões relacionadas ao envelhecimento. As facetas adicionais do WHOQOLOLD são: Funcionamento dos sentidos, Autonomia, Atividades passadas, presentes e futuras, Participação social, Morte e morrer e Intimidade (CHACHAMOVICH et al., 2008).

Em estudos posteriores ao desenvolvimento do WHOQOL-100 e WHOQOL-bref, foi constatado que o domínio Aspectos espirituais/Religião/Crenças pessoais nestes instrumentos era conceitualmente e empiricamente insuficiente. Frente a essa fragilidade, o Grupo WHOQOL pautou-se no desenvolvimento de um estudo para avaliar a forma com a qual o domínio em exame está relacionado com a QV (THE WHOQOL-SRPB GROUP, 2006).

O WHOQOL-SRPB não é um instrumento para avaliar a espiritualidade, mas sim, um instrumento que contempla, de forma ampliada, o constructo Aspectos espirituais/Religião/Crenças 
pessoais, representado no WHOQOL-100 e WHOQOL-bref de forma reduzida (THE WHOQOLSRPB GROUP, 2006).

Nessa perspectiva, o WHOQOL-SRPB suplementa o WHOQOL-100 com oito facetas adicionais ao domínio Aspectos espirituais/Religião/Crenças pessoais. Os demais domínios do WHOQOL-100 permanecem inalterados. As facetas adicionais do WHOQOL-SRPB são: Conexão com o ser ou força espiritual, Sentido na vida, Admiração, Totalidade e integração, Força espiritual, Paz interior, Esperança e otimismo e Fé.

$\mathrm{Na}$ tentativa de mensurar a QV de adultos portadores de deficiência física e/ou intelectual, recentemente fora desenvolvido um módulo adicional ao WHOQOL-bref direcionado para tal público. O WHOQOL-DIS é composto por 13 questões: uma geral - contemplando o impacto da deficiência - e 12 específicas, contemplando os seguintes aspectos: Discriminação, Proteção, Perspectivas futuras, Controle da vida, Tomada de decisões, Autonomia, Comunicação, Aceitação social, Respeito, Interação na sociedade, Inclusão social e Capacidade pessoal (POWER et al., 2010).

Fora desenvolvida, também, uma versão do WHOQOL-DIS direcionado para os responsáveis pelos cuidados da pessoa portadora da deficiência intelectual. A versão do WHOQOLDIS direcionada para deficientes intelectuais possui uma escala de resposta diferenciada dos demais instrumentos WHOQOL, sendo esta do tipo Likert de três pontos. Nesse caso, o WHOQOL-bref também deve ser aplicado com a adaptação para tal escala.

Sob a mesma justificativa que instigou o desenvolvimento do WHOQOL-SRPB - a insuficiência das questões para avaliar um determinado aspecto da $\mathrm{QV}$-, fora desenvolvido o instrumento WHOQOL-Pain.

Tal qual o WHOQOL-OLD, o WHOQOL-Pain é constituído pelo WHOQOL-100 acrescido do módulo adicional direcionado às pessoas que sofrem de dor crônica. Este foi desenvolvido pelo Centro de Estudo de Qualidade de Vida da Universidade de Bath, na Inglaterra. Não foi desenvolvido paralelamente em outros centros nem traduzido para outros idiomas. O domínio Dor e desconforto é composto por 16 questões seccionadas em quatro facetas: Alívio da dor, Raiva e frustração, Vulnerabilidade/medo/preocupação e Incertezas (MASON; SKEVINGTON; OSBORN, 2010).

\section{Considerações finais}

Transcorrido mais de uma década desde o surgimento do WHOQOL-100 e WHOQOL-bref, estes permanecem sendo utilizados em grande escala. Atualmente os instrumentos WHOQOL são disponibilizados em mais de 50 idiomas.

O rigor metodológico utilizado no processo de construção e validação dos instrumentos WHOQOL possibilita a obtenção de características psicométricas satisfatórias, perfazendo com que os resultados das aplicações de tais instrumentos sejam válidos, consistentes e fidedignos à população estudada.

$\mathrm{Na}$ conferência anual de 2010 da Sociedade Internacional para Pesquisas em Qualidade de Vida (ISOQOL), fora explicitado que a versão abreviada do WHOQOL-SRPB está em desenvolvimento, e que, futuramente, será construída a versão abreviada do WHOQOL-Pain (INTERNATIONAL SOCIETY FOR QUALITY OF LIFE RESEARCH, 2010). Segue em andamento, também, o projeto para a construção da versão abreviada do WHOQOL-OLD (FANG et al., 2012), e, recentemente, foi anunciada a versão preliminar da variante abreviada do WHOQOL-SRPB, o WHOQOL-SRPB-bref (SKEVINGTON; GUNSON; O'CONNELL, 2012).

A prospecção é de que novos instrumentos WHOQOL sejam criados. Até o momento foram desenvolvidos 12 instrumentos. Independentemente do surgimento de outros instrumentos, é pertinente ressaltar que a OMS proferiu, e, continua proferindo, por meio dos instrumentos WHOQOL, uma contribuição ímpar na promoção da QV. 


\section{Referências}

AUSTRALIAN WHOQOL FIELD CENTRE. Use the World Health Organization Quality of Life (WHOQOL) Instruments. Centre for Participant Report of University of Melbourne, Melbourne, 2006. Department of Psychiatry. Disponível em: <http://www.psychiatry.unimelb.edu.au/centres-units/cpro/whoqol/use-whoqol.html>. Acesso em 23 abr. 2012.

CHACHAMOVICH, E. et al. Desenvolvimento do instrumento WHOQOL-OLD. In: FLECK, M. P. A. et al. A avaliação de qualidade de vida: guia para profissionais da saúde. Porto Alegre: Artmed, 2008. p. 102-111.

FANG, J. et al. Development of short versions for the WHOQOL-OLD module. The Gerontologist, Oxford, v. 52, n. 1, p. 66-78, Jan./Feb. 2012.

FLECK, M. P. A. et al. Desenvolvimento da versão em português do instrumento de avaliação de qualidade de vida da OMS (WHOQOL-100). Revista Brasileira de Psiquiatria, São Paulo, v. 21, n.1, p.19-28, jan./mar. 1999.

GRUPO WHOQOL. Versão em português dos instrumentos de avaliação da qualidade de vida (WHOQOL). Faculdade de Medicina da UFRGS, 1998. Departamento de Psiquiatria. Disponível em: <http://www.ufrgs.br/psiq/whoqol1.html>. Acesso em: 13 abr. 2008.

INTERNATIONAL SOCIETY FOR QUALITY OF LIFE RESEARCH. 2010 conference abstracts. Quality of Life Research, Amsterdam, v. 19, n. Supplement 1, p. 1-144, Oct. 2010.

JIROJANAKUL, P.; SKEVINGTON, S. M.; HUDSON, J. Predicting young children's quality of life. Social Science and Medicine, New York, v. 57, n. 7, p. 1277-1288, Oct. 2003.

LUZNY, J. Quality of life in mentally ill people. In: UEHARA, T. (Org.). Psychiatric disorders: worldwide advances. Rijeka: Intech, 2011, p. 151-162.

MASON, V. L.; SKEVINGTON, S. M.; OSBORN, M. Assessing the properties of the WHOQOLpain: quality of life of chronic low back pain patients during treatment. The Clinical Journal of Pain, Philadelphia, v. 26, n. 7, p. 583-592, Sep. 2010.

O'CONNELL, K. et al. WHOQOL-HIV for quality of life assessment among people living with HIV and AIDS: results from a field test. AIDS Care, Abingdon, v. 16, n. 7, p. 882-889, Oct. 2004.

POWER, M. J. Development of a common instrument for quality of life. In: NOSIKOV, A.; GUDEX, A. (Org.). EUROHIS: developing common instruments for health surveys. 1. ed., Amsterdam: IOS Press, 2003. p. 145-163.

POWER, M. J et al. Development of the WHOQOL-Old module. Quality of Life Research, Amsterdam, v. 14, n. 10, p. 2197-2214, 2005.

POWER, M. J. et al. Development of the WHOQOL disabilities module, Quality of Life Research, Amsterdam, v. 19, n. 4, p. 571-584, may 2010.

SKEVINGTON, S. M.; GUNSON, K. S.; O'CONNELL, K. A. Introducing the WHOQOL-SRPB BREF: developing a short-form instrument for assessing spiritual, religious and personal beliefs within quality of life. Quality of Life Research, Amsterdam, v. 22, n. 5, p. 1073-1083, jun. 2013. 
THE WHOQOL GROUP. WHOQOL-bref: introduction, administration, scoring and generic version of assessment. Geneva: World Health Organization, 1996.

THE WHOQOL-HIV GROUP. WHOQOL-HIV instrument user's manual. Geneva: World Health Organization, 2002.

THE WHOQOL-SRPB GROUP. A cross-cultural study of spirituality, religion, and personal beliefs as components of quality of life. Social Science and Medicine, New York, v. 62, n. 6, p. 14861497, mar. 2006. 